\title{
Correction: Urban-rural residence and birth defects prevalence in Texas: a phenome-wide association study
}

Elisa Benavides, Philip J. Lupo, Miranda Sosa, Kristina W. Whitworth, Mark A. Canfield, Peter H. Langlois and Jeremy M. Schraw

(c) The Author(s), under exclusive licence to the International Pediatric Research Foundation, Inc 2021

Pediatric Research (2022) 91:1625; https://doi.org/10.1038/s41390-021-01737-7

Correction to: Pediatric Research https://doi.org/10.1038/s41390021-01700-6, published online 16 August 2021

The captions of Figs. 1 and 2 were inadvertently reversed in this article. The name of first author given in reference 10 has been changed from Long, $\mathrm{L}$ to $\mathrm{Li}, \mathrm{L}$. The original article has been corrected.

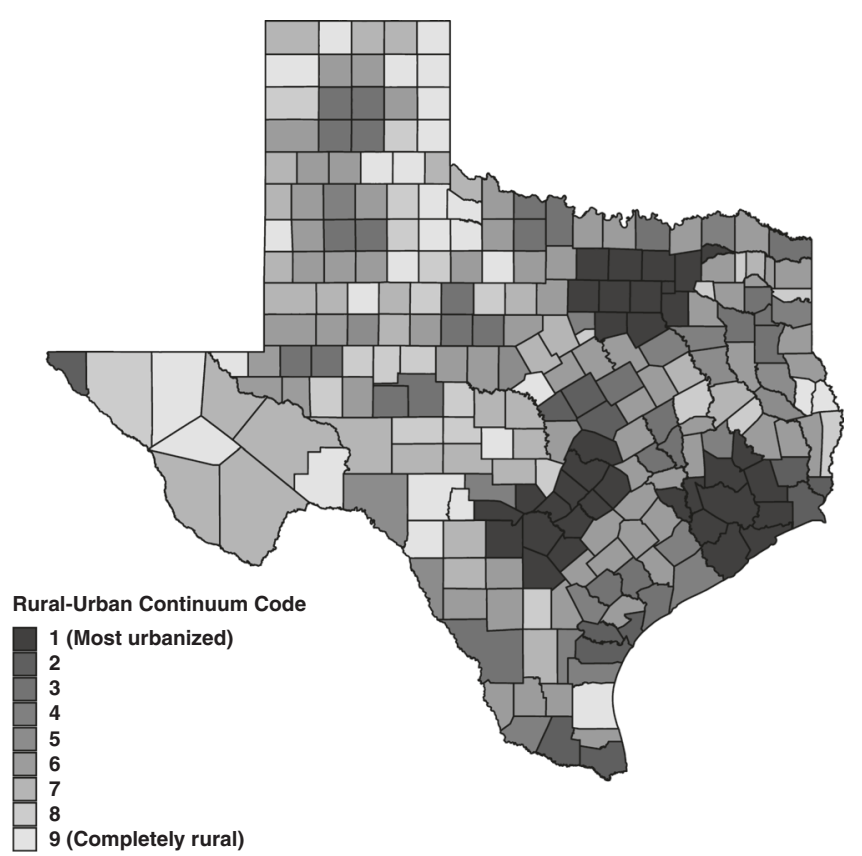

Fig. 12003 United States Department of Agriculture Economic Research Service Rural-Urban Continuum Codes (RUCC) for Texas counties. Lower scores (darker colors) indicate a greater degree of urbanization. In dichotomous analyses of urban vs. rural residence, urban counties were defined as those with RUCC $\leq 3$ and rural counties were defined as those with RUCC $\geq 4$.

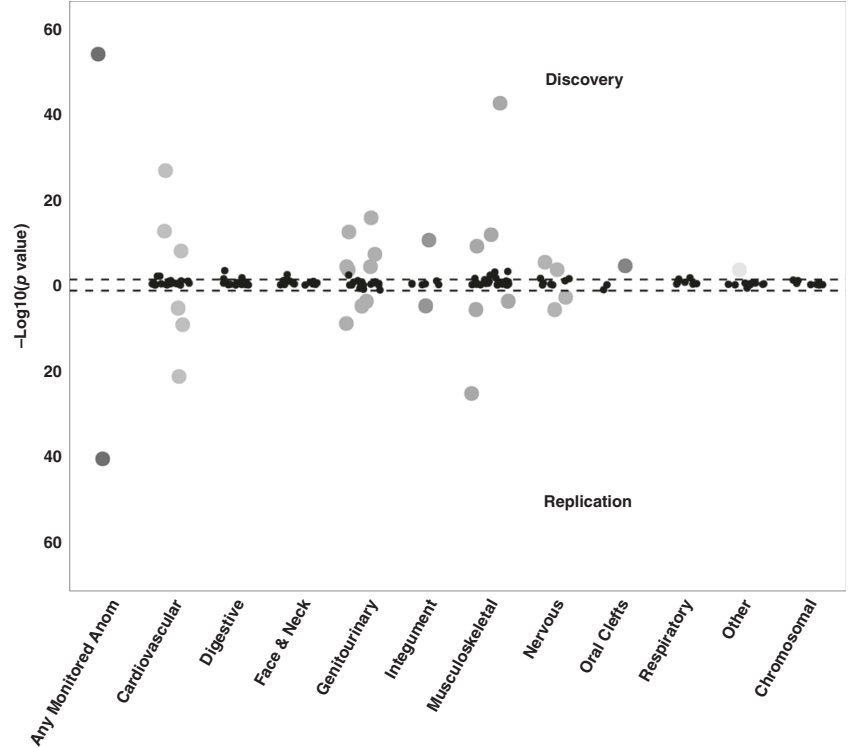

Fig. 2 Associations between urban-rural residence and birth defects. Birth defects associated with urban-rural residence at $p<3.55 \times 10-4$ in discovery (upper panel; $n=18$ ) were retested in replication (Fig. 2, lower panel). Those associated with urban-rural residence in replication at $p<0.05$ were declared significant $(n=13)$. 